\title{
SwissDRG: 3 ans et déjà la routine?
}

\section{Pierre-François Cuénoud}

Dr, vice-président de la FMH, responsable du département Tarifs et économie de la santé pour les médecins hospitaliers

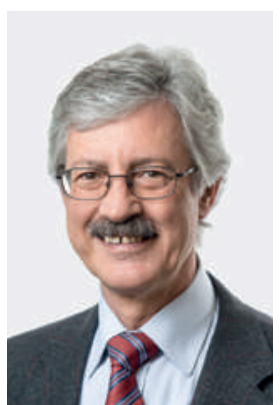

La structure tarifaire SwissDRG, avec plus de 1100 jours à son actif, en est déjà à sa 4 eversion. La volonté des partenaires, déterminés à insuffler une dynamique suffisante pour tendre rapidement à une bonne représentativité des prestations hospitalières stationnaires, a été respectée. Grâce à l'excellent travail du Case Mix Office (CMO), l'ambition d'élaborer puis entretenir un système de tarification helvétique particulier s'est avérée réaliste. La réactivité de celui-ci est bonne, avec des variations de poids relatifs de coût pouvant atteindre 30\% d'une version à l'autre.

Certes, tout n'est pas encore parfait. En particulier, les séjours générant des coûts très élevés restent une charge pour les hôpitaux à vocation centrale. L'évolution du climat politique et la sensibilité populaire actuellement très favorables aux missions générales de santé publique, se reflètent également indirectement sur la structure tarifaire hospitalière, les prestations de base ayant été graduellement valorisées au détriment de certains actes plus techniques et complexes. Ce phénomène fait manifestement reculer l'horizon d'un système suffisamment affiné pour qu'une rétribution correcte des prestations puisse être envisagée sans devoir différencier les taux de base par catégorie d'établissement.

Le passage à SwissDRG a été réussi. Tout n'est pas encore parfait, les séjours générant des coûts très élevés restent une charge pour les hôpitaux à vocation centrale.

Pour les patients, ces trois années de forfaits par cas généralisés n'ont pas entraîné de modification notoire. En revanche, la situation est souvent difficile pour les directions hospitalières. En effet, les négociations avec les assureurs aboutissent à des taux de base dégressifs, contraignant les décideurs à renoncer à certaines prestations pour conserver l'équilibre financier. Afin de maintenir un service sanitaire adé- quat au profit de leur population, les cantons doivent assumer les prestations d'intérêt général: certains n'en accordent aucune, condamnant leurs hôpitaux à maigrir pour survivre; d'autres font au contraire preuve d'une grande générosité, arrosant leurs établissements publics de centaines de millions chaque année ou leur accordant des crédits spéciaux pour le renouvellement de leurs infrastructures. Il en ressort une grande inégalité de traitement entre tous les intervenants du pays.

Bien que confrontés à une bureaucratisation croissante, les médecins hospitaliers ne négligent en rien leurs tâches cliniques.

Bien que confrontés à une bureaucratisation croissante, les médecins hospitaliers ne négligent en rien leurs tâches cliniques. On observe cependant un changement progressif des sensibilités. Alors qu'il y a 10 ans, un médecin cadre sur 20 disait s'intéresser au contexte économique, un tiers d'entre eux déclarent aujourd'hui s'impliquer activement dans la gestion de leur établissement, la plupart ayant suivi une formation managériale postgrade. Par ailleurs, de plus en plus de collègues quittent leur activité clinique pour se consacrer à de nouvelles professions strictement administratives, telles que le «management des itinéraires patients» récemment créé. L'équivalent d'une volée de finalistes a ainsi été perdu pour le travail auprès des patients en une décennie, en raison de ces transferts.

De manière générale, le passage à SwissDRG a été réussi. Par son engagement critique, la FMH œuvre constamment à l'amélioration de la structure tarifaire. D'autre part, elle assure le lien entre les utilisateurs médicaux et les instances faîtières. Elle garantit ainsi que le corps médical puisse garder une influence prépondérante sur notre système de santé hospitalier, trop évolutif pour tomber dans la routine. 